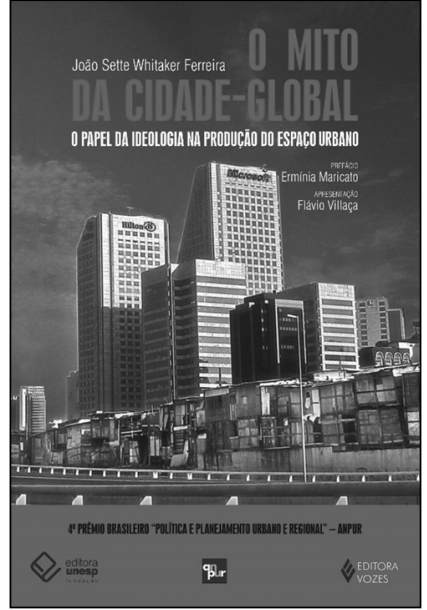

João Sette Withaker Ferreira

\section{El Mito de la Ciudad Global: el papel de la ideología en la produc- ción del espacio urbano}

Sao Paulo: Editora Vozes (2007)
Joao Sette Whitaker Ferreira, además de ser reconocido por su formación como arquitecto, economista, magíster en Ciencias Políticas y, por ser parte actualmente del cuerpo de profesores e investigadores de la Facultad de Arquitectura y Urbanismo de la Universidad de Sao Paulo, también se ha destacado por la tesis a con la que obtuvo su grado de Doctor en Estructuras Urbanas por esta misma universidad, titulada "Sao Paulo: el mito de la ciudad global"; premiada por la ANPUR ${ }^{1}$ en 2005 y que culmina en la publicación de este libro.

La hipótesis de ese trabajo es que, apoyándose en algunas constataciones de fenómenos reales -como el aumento de la oferta de edificios de comercio y servicios- pero poco significativos en cuanto a las estructuras de producción de la ciudad, se habría construido para Sao Paulo una interpretación de esos fenómenos, según la cual éstos serían un parámetro incontestable para la definición de las políticas urbanas de la ciudad. Se trataría de un proceso típicamente ideológico que busca universalizar la idea de que Sao Paulo es una "ciudad-global", generalizando la situación muy particular de determinadas islas en la ciudad a efectos de legitimar los intereses de aquellos que ganan con su consolidación.

Para dar soporte a ese análisis, el autor analiza las teorías sobre la "ciudad-global", tratando de destacar las principales características que las definen y verifica su presencia, o ausencia, en Sao Paulo. Es lo que resume Erminia Maricato en el Prefacio del libro: "El concepto de ciudad global hechizó a autores, profesores, editores, periodistas, consultores, como bien revela un gran número de tesis, articulos, reportajes, informes elaborados durante los años 90. Una mirada en la bibliografía $y$ en sus numerosas referencias del libro pone en evidencia esa afirmación. No obstante, por ninguna de sus concepciones, la clasificación se sostiene para Sao Paulo y consecuentemente para cualquier ciudad de Brasil. Es lo que João Whitaker demuestra con un trabajo detallado comparando conceptos, indicadores $y$ hechos en diversos actores" (Ferreira, 2007, p. 10).

\footnotetext{
Asociación Nacional de Posgrado e Investigación en Planificación Urbana y Regional
} 
Según Ferreira, la "ciudad-global" ha sido difundida - por los gobiernos, la prensa y las universidades- como el único modelo urbano capaz de garantizar la sobrevivencia de las ciudades en el "nuevo" contexto de "globalización de la economía". La ciudad-global se transforma en un objetivo, una especie de pasaporte para el ingreso en el Primer Mundo y, por lo tanto, en un modelo de sumisión absoluta al mercado, que si bien puede pasar por eficaz en las ciudades desarrolladas, es considerado desastroso por el autor en las grandes metrópolis periféricas, como Sao Paulo.

Es eso lo que aborda en este libro, en que trata de recopilar en tres de los cinco capítulos de la estructura principal del trabajo, las teorías sobre el concepto de ciudad mundial y global, los principales autores y obras, así como también sus características, transformadas en "recetas" y manejadas como discurso por los estratos más pudientes de Brasil y, más específicamente, por los que manejan el mercado inmobiliario. Además, en los dos capítulos restantes, Ferreira busca profundizar su análisis con bases empíricas para el caso de Sao Paulo y, en particular, para la región de la Marginal Pinheiros, promovidas como íconos de la "globalidad" brasileńa.

En el primer capítulo, titulado Sao Paulo, ciudad global: la construcción de un mito, el autor contrapone la explicación urbana de la globalidad a las verdaderas raíces de la desigualdad estructural brasileña, que se reflejan en sus ciudades desde el periodo colonial, pero con especial énfasis en el periodo de la llamada "industrialización con bajos sueldos", en la segunda mitad del siglo XX. Además, analiza el tema de la ideología como un instrumento por el cual las clases dominantes legitiman el discurso de su interpretación de la realidad, cuestión que ocurre en este caso, con el discurso de la "ciudad-global".

El concepto de ideología es tomado en su acepción marxista, que viene junto al de lucha de clases y de división social del trabajo. Ferreira, así, cree que tal visión de ideología se aplica a lo urbano una vez que la producción del espacio reproduce las dinámicas de lucha de clases en el territorio, en las disputas por el espacio y por la apropiación de su valoración.

En el segundo capítulo, Sao Paulo, ciudad global: la desconstrucción de un mito, así como en el quinto, titulado Las demandas muy locales, pocos globales, de la producción del espacio terciario en Sao Paulo: la máquina paulista del crecimiento, revisa cómo el concepto de ciudad global es distorsionado para el caso de Sao Paulo. A través del análisis de datos empíricos busca mostrar que la ciudad no presenta ninguno de los atributos típicos listados por los defensores de la teoría, como Sassen, para definir a la "ciudad global".

Esa investigación muestra, uno a uno, los principales argumentos constitutivos de lo que sería una "ciudad-global", aplicados a la realidad de la ciudad de Sao Paulo. Así, la ciudad no sería sede de empresas entre las mayores transnacionales; su aeropuerto tendría flujos proporcionalmente bajos comparados al tamańo de la ciudad (una de las cinco mayores metrópolis del mundo); tampoco ocurriría la supuesta transferencia del empleo industrial hacia el sector terciario, existiendo, en realidad, una enorme precarización del empleo, con una transferencia masiva hacia la informalidad, igual para el sector industrial todavía bien presente en la realidad paulista. 
Ferreira también muestra que no sería verdadera la idea tan difundida de que en la región estudiada de la "centralidad global" de la Avenida Berrini (área de la Marginal Pinheiros), existe una concentración de empresas del sector "terciario avanzado", dado que en verdad ellas se dispersan por toda la ciudad; por ello, este sector no vendría a ser tan importante en la economía local como lo hacen creer los defensores de la teoría de las "ciudades-globales".

Por otra parte, en el quinto capítulo muestra cómo, apoyándose en esa falsa realidad, las elites urbanas conseguirían canalizar recursos públicos para la construcción de "islas globalizadas" (centros de convenciones y otros mega-emprendimientos terciarios del mismo orden). Aunque promuevan su rol como pasaporte para el Primer Mundo, en realidad solamente desarrollarían una rápida valorización del suelo e inmobiliaria, generando ganancias extraordinarias y exacerbando la concentración del dinero sólo en áreas urbanas ultra-privilegiadas.

Para el autor, el objetivo de la difusión ideológica del mito de la "ciudad-global" es la disputa, por parte de los distintos grupos del mercado inmobiliario -que el autor llama "frentes inmobiliarios" - en torno a fondos públicos para infraestructura urbana, capaces de valorizar la tierra y generar ganancias extraordinarias. La tan llamada "internacionalización” del sector sería, como muestra el autor, otro mito, que tan solo se vuelve parcialmente real en 2006, muchos años después de la construcción de la centralidad-global estudiada y, además, de forma tímida (con la entrada en la bolsa de las constructoras, atrayendo, así, inversiones externas que no ocurrían en la década de los 90 e inicio de los 2000).

Se identifica, entonces, un fenómeno de economic growth machine ("máquina del crecimiento urbano") en el que los grupos inmobiliarios dominantes ejercerían una verdadera disputa, y conseguirían, así, focalizar hacia ellos grandes sumas de dinero público, siempre bajo el justificativo -rápidamente legitimado por la prensa, por las universidades y por los gobiernos- de que tal priorización de inversiones sería necesaria para la "condición global” de la ciudad, lo que generaría fantasiosas "sinergias urbanas" que, en realidad, nunca habrían ocurrido.

La idea de economic growth machine, de autoría de los norteamericanos Logan y Moloch, es analizada más profundamente en el cuarto capítulo, Reestructuración productiva y producción del espacio urbano en la teoría de la máquina del crecimiento, en el cual el autor se detiene en otras formas posibles de definir una "ciudad-global", distintas a la propuesta por la teoría en boga. Una vez más, analizando casos de diversas partes del mundo, el autor muestra cómo en Sao Paulo no ocurrieron fenómenos como la transferencia de capitales extranjeros para el mercado inmobiliario local (como en Tailandia), ni un proceso de liberalización del mercado inmobiliario.

A partir de allí, se propone a la teoría de la máquina del crecimiento como una matriz explicativa para la ciudad de Sao Paulo, siempre que sea objeto de algunos ajustes, pues en USA los emprendedores en coalición, para hacer andar a la "máquina del crecimiento", serían aquellos que sacan de la tierra su valor de cambio (emprendedores, constructores, políticos y empresas), los grupos que a ellos se confrontarían serían aquellos que sacan de la tierra su valor de uso, a saber, inquilinos y propietarios viviendo en sus casas; sin embargo, para Ferreira, en Brasil la disputa es un poco distinta, ya que siendo la ciudad segregada, la disputa no se daría entre 
usuarios y emprendedores, sino entre incluidos y excluidos del sistema económico y de la ciudad, lo que caracterizaría la exacerbación de la polarización social brasilera.

Sin embargo, sería imposible tratar la temática de la "ciudad-global" si no se analiza más detenidamente el propio fenómeno de la globalización, lo que el autor hace en el tercer capítulo titulado Globalización, ideología y pensamiento urbano. El término ciudad mundial fue utilizado por Patrick Gueddes ya en 1915, y fue retomado por Peter Hall en 1966, en "World Cities". Ferreira reconoce que la idea principal correspondería al papel ejercido por las principales ciudades mercantiles desde la Antigüedad: "en la organización económica mundial, ciertas ciudades, más que otras, tendrian más dominio estratégico por su posición geográfica, su potencial comercial, su desempeño económico, su influencia politica y asi por delante" (Ferreira, 2007, p. 20).

De acuerdo a teorías más contemporáneas, las transformaciones económicas ocurridas en el mundo a partir de los años 70 habrían alterado la función y las formas de la organización y la estructuración de las principales ciudades mundiales, en especial aquellas más destacadas en el sistema económico global. Se crea, entonces, una dualidad compleja, donde la economía se dispersaría en el espacio y, al mismo tiempo, consolidaría su integración a escala planetaria. Para Sassen, "esa combinación de dispersión geográfica y de integración mundial creó un nuevo papel estratégico para las grandes ciudades” (Sassen en Ferreira, 1996, p. 32).

Ahora, aunque los distintos enfoques teóricos de la planificación estratégica y de las "ciudadesglobales" puedan ser divergentes en algunos puntos, ya sea una visión más académica y científica como Sassen, o más instrumental como Borja y Castells, lo que el autor destaca es que ninguna de ellas está exenta de un fuerte contenido ideológico. Por eso, afirma que construyen su diagnóstico de la "ciudad-global" como un paradigma de lo que debería ser la ciudad en el escenario propio de la construcción ideológica de la globalización.

Según Ferreira, se trata de un típico proceso ideológico que busca universalizar la idea de que la "ciudad global" de interés para todos, legitimando una "máquina de crecimiento urbano" que relegaría los problemas sociales a segundo plano, para satisfacer intereses muy específicos de los sectores dominantes más tradicionales del mercado inmobiliario.

Por lo tanto, lo que el autor trata de concluir es que la globalidad de la ciudad de Sao Paulo -así como cualquier ciudad brasileña- es parcial, al ser aplicable solamente a un parte de la ciudad y, además, incompatible con la disminución de las significativas desigualdades sociales y espaciales.

Lo que parece interesante en el análisis del autor es verificar si el modelo ideológico que denuncia se aplica a Sao Paulo y aparentemente a otras ciudades brasileñas, y también a muchas de las grandes capitales latinoamericanas, donde la búsqueda de la "globalidad" y la construcción de distritos de negocios - generalmente con uso de inversiones públicas para la construcción de infraestructura necesaria, con grandes ganancias para el mercado inmobiliario- es la tónica de esta fase de modernización, en detrimento de inversiones mucho más urgentes en las periferias pobres de la ciudad. 
En cuanto a su relación a Santiago, este libro parece ser una importante referencia para un análisis profundizado sobre las características y la veracidad del discurso de "ciudad-global" entregado a los grandes centros urbanos como este, pudiendo hacerse cierta comparación de metodología de estudio sobre casos igualmente calificados como "nuevos distritos centrales de negocio", como lo es el sector de "Sanhatan", el cual posee muchas de las características semejantes al sector paulista de Berrini y que, además, los conflictos parecen encaminarse en la misma dirección.

Conforme la definición de Ferreira sobre el sector Berrini, parecería que estuviéramos leyendo el futuro del CBD chileno: “(...) el boom de edificios terciarios llevó mucha gente a trabajar en la región, sin que la infraestructura de servicios (restaurantes, estacionamientos, comercio) [y también la malla vial] diera cuenta del nuevo flujo de usuarios. La dificultad de accesos se transformó en un problema para las empresas interesadas en instalarse en el área, y en la primera década de este siglo aumentó visiblemente la vacancia en la región.” (Ferreira, 2007, p. 38).

Vanessa Prado*

* Arquitecta y urbanista por la Facultad de Arquitectura y Urbanismo de la Universidad de Sao Paulo, Brasil. Candidata a Magíster de Desarrollo Urbano por el Instituto de Estudios Urbanos y Territoriales de la Pontificia Universidad Católica de Chile.

Correspondencia: vanyfau@gmail.com 